\title{
Desenvolvimento de métodos para detecção automática do glaucoma
}

\author{
Antônio Sousa Vieira de Carvalho Júnior ${ }^{1}$, Antônio Oseas de Carvalho Filho ${ }^{1}$, \\ Alcilene de Sousa ${ }^{1}$, Patricia Medyna Lauritzen de Lucena Drumond ${ }^{1}$, \\ Patricia da Silva Barros ${ }^{1}$ \\ ${ }^{1}$ Campus Senador Helvídio Nunes de Barros - Universidade Federal do Piauí (UFPI) \\ Picos, PI - Brazil \\ \{junior_sousa95, patymedy\}@hotmail.com, \{antoniooseas, alcileneluzsousa, \\ petibarrosthe\}@gmail.com
}

\begin{abstract}
Glaucoma is an ocular disease developed by the increase of the intraocular pressure that causes the progressive loss of the visual field. Because the condition is asymptomatic, it is of great importance that the disease be identified in the early stages thus facilitating treatment. The Digital Image Processing in conjunction with computational techniques has been developing methods for automatic detection of glaucoma. In this way, a comparison was made between four methods developed using the Otsu and k-means algorithms. In the first stage, optical segmentation was made. In the second stage, the extraction of characteristics based on the phylogenetic diversity indexes was used, and finally the classification using the WEKA, obtaining the best result applying the Otsu with sensitivity of $97.5 \%$ Specificity of $100.0 \%$ and an accuracy of $97.9 \%$.
\end{abstract}

Resumo. O glaucoma é uma doença ocular desenvolvida pelo aumento da pressão intraocular que ocasiona a perda progressiva do campo visual. Devido à patologia ser assintomática, é de grande importância que a doença seja identificada nos estágios iniciais facilitando assim o tratamento. O Processamento Digital de Imagens em conjunto com técnicas computacionais vem desenvolvendo métodos para detecção automática do glaucoma. Desta forma, foi realizada uma comparação entre quatro métodos desenvolvidos utilizando os algoritmos de Otsu e k-means. Na primeira etapa foi feita a segmentação do disco óptico, na segunda etapa foi utilizada a extração de características baseada nos índices de diversidade filogenética e por fim a classificação utilizando o WEKA, obtendo o melhor resultado aplicando o Otsu com sensibilidade de 97,5\%, especificidade de 100,0\% e uma acurácia de 97,9\%.

\section{Introdução}

O glaucoma é uma doença crônico-degenerativa, definida como uma neuropatia óptica multifatorial que provoca inúmeras afecções oculares em células da retina e do nervo óptico [Mello et al. 2016]. Trata-se de uma patologia que, a princípio, não apresenta sintomas, de forma que seu prognóstico está relacionado à detecção e ao tratamento precoce.

As doenças oculares afetam grande parte da população mundial. Dados levantados pela Organização Mundial da Saúde (OMS) revelaram que o glaucoma é a segunda maior causa de cegueira irreversível no mundo [Resnikoff et al. 2004]. 
A Pressão Intraocular (PIO) é o principal fator de desenvolvimento do glaucoma. Este acontece devido ao canal de drenagem do líquido humor aquoso ser subitamente obstruído, originando assim no aumento da PIO ocasionando a morte das células da retina e do nervo óptico.

A detecção do glaucoma nos estágios iniciais aumenta a possibilidade de obter um melhor tratamento. Este diagnóstico pode ser realizado através de exames periódicos, tais como a Tomografia de Coerência Óptica e Tomografia Retiniana Heidelberg ou por meio dos métodos computacionais com análises de imagens da retina. Perante a dificuldade para identificar a patologia, o Processamento Digital de Imagens (PDI) está desenvolvendo um papel importante na detecção automática do glaucoma através de métodos que apresentam um menor custo e possibilitam auxiliar o oftalmologista com outras maneiras de reconhecer e classificar o tipo do glaucoma.

Esse trabalho tem como objetivo apresentar o desenvolvimento de métodos para detecção automática do glaucoma utilizando os algoritmos Otsu e k-means. A princípio, foi realizada a segmentação do Disco Óptico (DO) para ser efetuada a extração de características com descritores de textura baseados em Índices de Diversidade Filogenética (IDF) e, por fim, classificar os atributos de modo a verificar quais dos métodos obteve melhor desempenho na detecção automática do glaucoma.

\section{Trabalhos Relacionados}

Esta seção é voltada para abordagens de alguns trabalhos desenvolvidos com outros métodos para detecção automática do glaucoma, de modo a analisar cada metodologia empregada.

No trabalho de Santos e Veras (2012) foi realizada a segmentação do DO utilizando o algoritmo transformada wavalet Gabor em uma base de domínio público intitulada Drive com 40 imagens da retina. Para avaliar a eficácia do método proposto foi feita a comparação de dois métodos e foi adotada uma métrica de sucesso e falha obtendo um resultado com sucesso de 35 imagens resultando em $87.5 \%$ de acerto.

Em Claro (2015) a proposta do trabalho tinha como finalidade usar imagens da retina para a detecção automática do glaucoma através da extração e classificação. A base de imagens utilizada foi a RIM-ONE, que possui 169 imagens fornecidas por diversos especialistas, onde apresentam imagens de olhos saudáveis e com vários níveis de glaucoma. A princípio segmentou-se a região do DO utilizando uma máscara fornecida pela base, em seguida foram extraídas as características utilizando as cores e a entropia. Após isso, houve uma classificação obtendo-se o melhor resultado no classificador Randon Forest, com 93,67\% e uma Acurácia e Kappa de 0,83.

Em Santos et al. (2015) a metodologia trabalhou apenas com o canal vermelho para melhorar a qualidade da segmentação. No estudo foram usadas três bases diferente: RIM-ONE, DRISHTI-GS e DRIONS-DB. Na segmentação foi necessário encontrar o centro do DO para que pudesse circular a região que pertencia ao DO onde acontece o glaucoma. Quando comparada a segmentação das três bases a que apresentou os melhores resultados foi a RIM-ONE obtendo uma acurácia de $83 \%$.

No estudo de Pinheiro et al. (2015), a proposta do trabalho foi segmentar a região de interesse utilizando o canal Vermelho (Red), Verde (Green) e Azul (Blue), em conjunto 
com algoritmo K-means separando em 5 clusters para definir a região do DO e melhorar a segmentação. Na validação dos resultados foi utilizado o classificador F-scores chegando a uma porcentagem de acerto em $91,75 \%$.

Entre os trabalhos relacionados, observa-se a necessidade de melhorar os resultados em Pinheiro et al. (2015), pois as manchas ao redor dos pixels do DO não foram retiradas, visto que ocorreu uma má segmentação de forma a modificar os resultados. Já em Santos e Veras (2012) a utilização de poucas imagens atrapalha na validação. A proposta desse trabalho é apresentar o desenvolvimento de dois métodos automáticos para a detecção do glaucoma usando os algoritmos de Otsu e k-means, para melhores resultados que os encontrados na literatura, com finalidade de auxiliar os médicos com resultados significativos, facilitando o diagnóstico precoce do glaucoma.

\section{Materiais e métodos}

A metodologia proposta neste trabalho teve como objetivo utilizar segmentação do DO para extrair as características com descritores de texturas baseados em IDF, aplicando classificadores para detecção automática do glaucoma. Durante o desenvolvimento, a metodologia foi dividida em 5 etapas, como ilustrado no fluxograma da Figura 1.

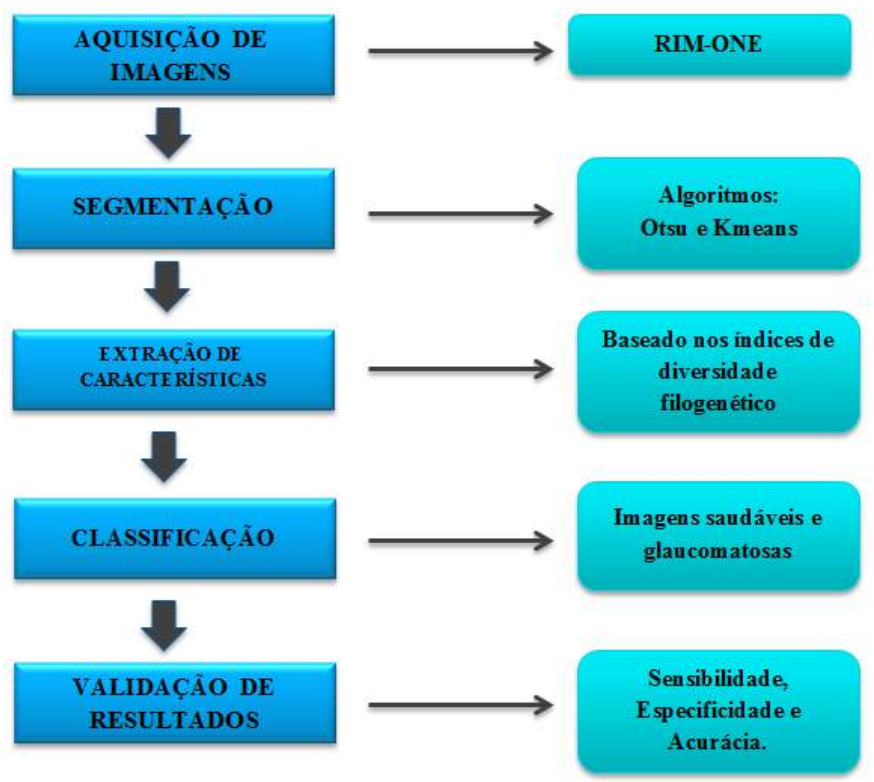

Figura 1. Fluxograma de execução do projeto.

\subsection{Aquisição de Imagens}

No estudo foi utilizada a base RIM-ONE que contém 169 imagens da retina e, como a base apresenta algumas imagens com outros tipos de lesões, foram aproveitadas no método proposto 144 imagens, sendo 26 imagens que apresentam glaucoma em vários estágios e 118 são de olhos saudáveis, fornecida por três hospitais: Hospital Universitário de Canarias, Hospital Clínico San Carlos e Hospital Universitário Miguel Servet.

\subsection{Segmentação}

$\mathrm{Na}$ etapa de segmentação foram usados os algoritmos de Otsu e k-means, onde o Otsu busca determinar um limiar de forma a maximizar a variância entre classes. A operação 
de limiarização consiste do particionamento dos pixels de uma imagem com $n$ níveis de cinza em duas classes, objeto e o fundo[Bertholdo 2007].

O Otsu analisa o histograma de cores presente na imagem, de modo a encontrar o melhor limiar para separar o fundo da região de interesse onde o glaucoma se apresenta.

O k-means é um algoritmo de aprendizado não-supervisionado que procura formar grupos com base nos valores médios da distância euclidiana entre os atributos das diversas áreas em um conjunto de dados [Andrade et al. 2016].

O k-means separa uma imagem em 2 classes. Nesse caso o $K$ é igual a 2, onde $K$ representa a quantidade de centróides criados para auxílio no encontro das semelhanças dos dados. Uma forma de fazer isso é inserindo os centróides aleatoriamente, para que as iterações possam dividir as classes. Com finalidade de obter outros resultados também foi implementada a região circular em conjunto com o k-means.

Para identificar o glaucoma é necessário isolar a região de interesse onde a patologia se apresenta, segundo os oftalmologistas o glaucoma se manifesta na região de DO. Na tentativa de obter melhores resultados traçou-se uma circunferência em torno da região segmentada pelos algoritmos de Otsu e K-means. Desenhou-se um plano cartesiano representativo ilustrado na Figura 2, onde é necessário encontrar o valor do menor X, maior $\mathrm{X}$, menor $\mathrm{Y}$ e maior $\mathrm{Y}$ de modo a calcular o diâmetro, o qual é adquirido quando encontrando a maior distância entre dois pontos, calculando-se, assim, o raio para delinear a circunferência de tudo que esteja dentro do raio.

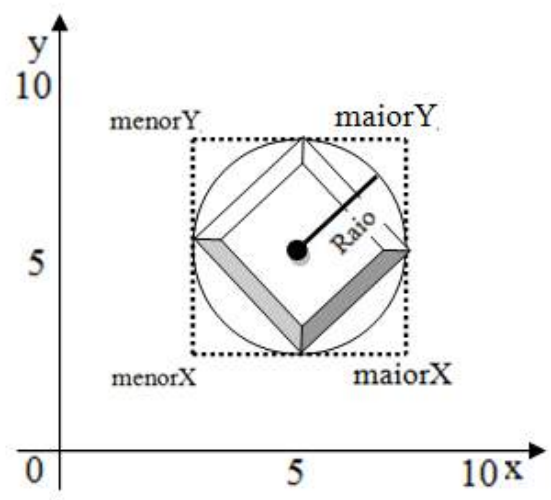

Figura 2. Ilustração do Plano Cartesiano.

A Figura 3 mostra três imagens, onde (a), é uma imagem glaucomatosa da retina, (b) é aplicado o algoritmo de Otsu para a segmentação e (c), utiliza-se o Otsu com a circunferência. 


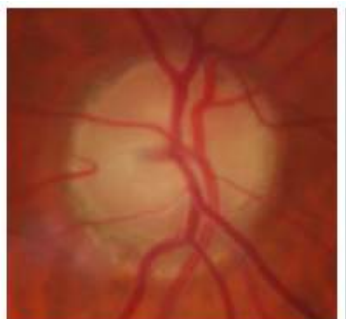

(a)

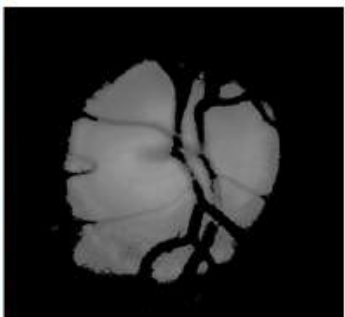

(b)

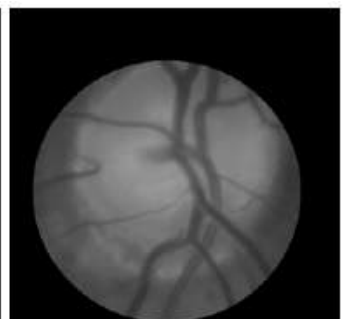

(c)

Figura 3. Imagem glaucomatosa da retina (a), Segmentação aplicando Otsu (b), Segmentação aplicando de Otsu com a região circular (c).

A Figura 4 mostra três imagens, onde (a) é uma imagem glaucomatosa da retina, (b) aplicou-se o algoritmo k-means para a segmentação, e (c) utilizou-se o k-means com a circunferência para segmentar a região de interesse.

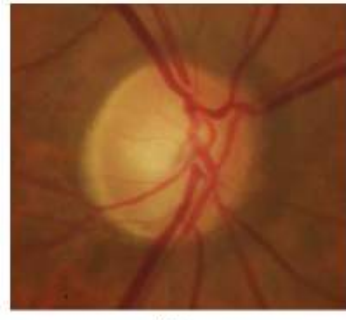

(a)

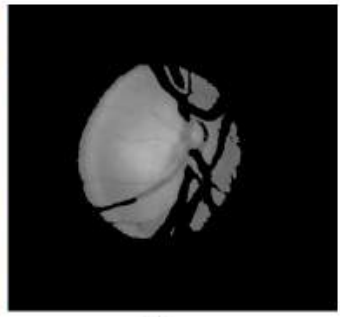

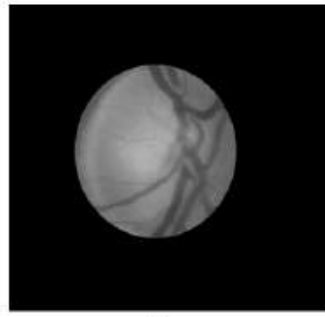

(c)

Figura 4. Imagem glaucomatosa da retina (a), Segmentação aplicando k-means (b), Segmentação aplicando k-means com a região circular (c).

\subsection{Extração de Características}

É possível caracterizar uma área contida em uma imagem por meio de extração de características estatísticas aplicando a análise de textura. Esta é uma abordagem natural que representa uma medida onde tal propriedade pode interpretar diversas informações visuais. Os descritores de textura contêm informações sobre a distribuição espacial, variação de luminosidade, suavidade, rugosidade, regularidade e descreve o arranjo estrutural das superfícies e as relações entre os pixels próximos [Oliveira e Fernandes 2016].

Nesta etapa a extração de característica foi desenvolvida utilizando descritores de texturas baseados em IDF. Os índices de diversidade são aplicados na ecologia para medir a biodiversidade em um ecossistema. Com a compreensão da estrutura filogenética da comunidade, é possível construir generalizações amplamente manuseadas para entender os reais processos que alteram as estruturas das comunidades [Moreira 2014]. Os IDFs são métricas de grupamento em setores de uma área, de forma a estimar diversidade de uma população, na qual cada indivíduo pertence apenas a um grupo.

Nos IDFs Phylogenetic Diversity (PD) é calculado o somatório dos comprimentos dos ramos de cada classe e $B$ é o número de ramificação da árvore, $L i$ é comprimento do ramo e $A i$ é a abundância média de classes que compartilham ramos, como mostrada na Equação 1. 


$$
B X \frac{\sum_{i}^{B} L_{i} A_{i}}{\sum_{i}^{B} A_{i}}
$$

A Equação 2 mostra a representação do índice filogenético Sum of Phylogenetic Distances (SPD) que é a soma das distâncias filogenéticas entre cada par de classe e $d_{m n}$ é a distância entre as classes $m$ e $n, a_{m}$, abundância de classes $m, S$ é o numero de classes no conjunto focal.

$$
\left(\frac{S(S-1)}{2}\right) X \frac{\Sigma \Sigma m<n^{2} m n^{a} m^{a} n}{\Sigma \Sigma m<n^{a} m^{a} n}
$$

O Mean Nearest Neighbour Distance (MNND) trata-se da distância média do táxon mais próximo, onde $d_{m n}$ é a distância entre as espécies $m$ e $n, a_{m}$, é abundância de espécies $m$. A formula é mostrada na Equação 3.

$$
\sum_{m}^{S} \min \left(d_{m n}\right) a_{m}
$$

O índice diversidade Phylogenetic Species Variability (PSV) é a variabilidade de classes filogenéticas, que retrata o grau em que as classes em uma comunidade são filogeneticamente relacionadas, como mostrado na Equação 4, onde, o $\operatorname{tr} C$ corresponde a soma dos valores da diagonal de uma matriz $\sum C,{ }^{\prime} c$ e o somatório de todos os valores da matriz, $n$ é o numero de classes e ${ }^{\prime} c$ é a media dos elementos da diagonal de $C$.

$$
P S V=\frac{n t r C-\Sigma c}{n(n-1)}=1-{ }^{\prime} c
$$

O IDF Phylogenetic Species Richness (PSR) é a riqueza de classes e quantifica o número de classes em um grupo. O valor do PSR é encontrado multiplicando-se o número de classes n pela variabilidade da comunidade, conforme os parâmetros na Equação 5.

$$
P S R=n P S V
$$

Os IDFs foram utilizados para caracterizar a textura das regiões segmentadas, de modo, a extrair as características contidas em cada imagem segmentada pelos algoritmos Otsu e k-means.

\subsection{Classificação}

Após a extração das características com análise de textura baseados em IDF a próxima etapa é a classificação, que beneficia-se do framework WEKA já que o mesmo contém um acervo de algoritmos para aprendizado de máquina. Na classificação foram utilizados quatro classificadores J48, BayesNet, AttributeSelectedClassifier e DecisionTable, pelo fato de serem rigorosos em relação a presença de ruídos no processo de classificação para a detecção automática do glaucoma. Na literatura alguns trabalhos já os utilizaram e obtiveram bons resultados, manipulando os parâmetros com os valores padrões em conjunto com a validação cruzada de $k$-folds, sendo $\mathrm{k}=10$. Esse procedimento divide os atributos 
em 10 grupos, de forma a realizar o treino em 9 grupos e operar um grupo para testes. $\mathrm{Na}$ realização de 10 cruzamentos, os grupos de teste são mesclados e ao final é gerada uma média, onde se configura o resultado.

\subsection{Validação dos Resultados}

As métricas de avaliação dos resultados foram ilustradas em uma matriz de confusão baseado em quatro valores: Verdadeiro Positivo (VP), que é o número de imagens corretas classificadas como glaucomatosas; Falso Positivo (FP), onde valida o número de imagens classificadas como saudáveis sendo que são glaucomatosas, Falso Negativo (FN), que são as imagens classificadas como glaucomatosas, porém são saudáveis e o Verdadeiro Negativo (VN) que resulta no número de imagens classificadas como saudáveis.

Para avaliar os resultados a fim de descobrir qual o melhor método desenvolvido utilizou-se os índices estatísticos que são: Sensibilidade, Especificidade e Acurácia.

Sensibilidade (S), é a capacidade de um teste diagnóstico identificar os VP nos indivíduos doentes, como mostra na Equação 6.

$$
S=\frac{V P}{V P+F P}
$$

Especificidade (E), trata-se da capacidade de um teste diagnóstico identificar os VN nos indivíduos sadios, como mostra na Equação 7.

$$
E=\frac{V P}{V P+F N}
$$

E Acurácia (A), é a proporção de acertos, ou seja, o total VP e VN da amostra estudada, como mostra na Equação 8.

$$
A=\frac{V P+V N}{V P+F P+V N+F N}
$$

\section{Resultados}

Os resultados obtidos na classificação dos métodos desenvolvidos para a detecção automática do glaucoma são mostrados nas Tabelas 1, 2, 3, 4, 5 e 6.

A Tabela 1 apresenta os resultados obtidos aplicando o algoritmo de Otsu para a segmentação e os descritores de textura baseados em IDF.

Tabela 1. Resultados da classificação com Otsu.

\begin{tabular}{cccccccc}
\hline & FP & FN & VP & VN & Sensibilidade & Especificidade & Acurácia \\
\hline BayesNet & $\mathbf{3}$ & $\mathbf{1}$ & $\mathbf{1 1 5}$ & $\mathbf{2 5}$ & $\mathbf{9 9 , 1 \%}$ & $\mathbf{8 9 , 3 \%}$ & $\mathbf{9 7 , 2} \%$ \\
J48 & 2 & 1 & 116 & 25 & $99,1 \%$ & $92,6 \%$ & $97,9 \%$ \\
AttributeSelectedClassifier & 2 & 1 & 116 & 25 & $99,1 \%$ & $92,6 \%$ & $97,9 \%$ \\
DecisionTable & $\mathbf{2}$ & $\mathbf{0}$ & $\mathbf{1 1 6}$ & $\mathbf{2 6}$ & $\mathbf{1 0 0 , 0} \%$ & $\mathbf{9 2 , 9 \%}$ & $\mathbf{9 8 , 6 \%}$ \\
\hline
\end{tabular}


Entre os resultados obtidos da classificação mostrada na Tabela 1, o que obteve o melhor resultado foi o classificador DecisionTable, sendo que, das 144 imagens utilizadas, 142 foram consideradas corretas e 2 falsas, conseguindo uma sensibilidade de $100,0 \%$, especificidade de $92,9 \%$ e acurácia $98,6 \%$. O resultado menos significativo é do classificador BayesNet, que declarou 140 imagens como corretas e 4 falsas, alcançando uma sensibilidade de 99,1\%, Especificidade de 89,3\% e acurácia 97,2\%.

A Tabela 2 mostra os resultados adquiridos aplicando o algoritmo de Otsu com a região circular e os descritores de textura baseados em IDF.

Tabela 2. Resultados da classificação com Otsu e a região circular.

\begin{tabular}{cccccccc}
\hline & FP & FN & VP & VN & Sensibilidade & Especificidade & Acurácia \\
\hline BayesNet & 0 & 3 & 118 & 23 & $97,5 \%$ & $100,0 \%$ & $97,9 \%$ \\
J48 & $\mathbf{2}$ & $\mathbf{2}$ & $\mathbf{1 1 6}$ & $\mathbf{2 4}$ & $\mathbf{9 8 , 3} \%$ & $\mathbf{9 2 , 3} \%$ & $\mathbf{9 7 , 2} \%$ \\
AttributeSelectedClassifier & 2 & 2 & 116 & 24 & $\mathbf{9 8 , 3} \%$ & $\mathbf{9 2 , 3} \%$ & $\mathbf{9 7 , 2 \%}$ \\
DecisionTable & $\mathbf{0}$ & $\mathbf{3}$ & $\mathbf{1 1 8}$ & $\mathbf{2 3}$ & $\mathbf{9 7 , 5 \%}$ & $\mathbf{1 0 0 , 0} \%$ & $\mathbf{9 7 , 9 \%}$ \\
\hline
\end{tabular}

Entre os resultados atingidos na Tabela 2, o melhor resultado foi com DecisionTable, que das 144 imagens analisadas foram consideradas que 141 estariam corretas e 3 falsas, resultando em uma porcentagem de acerto com sensibilidade de 97,5\%, especificidade de $100,0 \%$ e uma acurácia com $97,9 \%$. O pior resultado entre os classificadores foi o $J 48$, onde considerou que 140 imagens estão corretas e 4 são falsas, chegando uma proporção do resultado com sensibilidade de $98,3 \%$, especificidade $92,3 \%$ e acurácia de $97,2 \%$.

A Tabela 3 apresenta os resultados da segmentação usando algoritmo K-means e os descritores de textura baseados em IDF.

Tabela 3. Resultados da classificação com o K-means.

\begin{tabular}{cccccccc}
\hline & FP & FN & VP & VN & Sensibilidade & Especificidade & Acurácia \\
\hline BayesNet & 19 & 1 & 99 & 25 & $99,0 \%$ & $57,8 \%$ & $86,2 \%$ \\
J48 & 7 & 7 & $\mathbf{1 1 1}$ & $\mathbf{1 9}$ & $\mathbf{9 4 , 1 \%}$ & $\mathbf{7 3 , 1 \%}$ & $\mathbf{9 0 , 3} \%$ \\
AttributeSelectedClassifier & 18 & 1 & 100 & $\mathbf{2 5}$ & $99,0 \%$ & $58,1 \%$ & $86,8 \%$ \\
DecisionTable & $\mathbf{1 9}$ & $\mathbf{1}$ & $\mathbf{9 9}$ & $\mathbf{2 5}$ & $\mathbf{9 9 , 0 \%}$ & $\mathbf{5 6 , 8 \%}$ & $\mathbf{8 6 , 1 \%}$ \\
\hline
\end{tabular}

Dos resultados exibidos na Tabela 3, o que apresentou resultado mais representativo foi o classificador $J 48$, analisando 144, sendo que, foram classificadas 130 imagens como corretas e 14 sendo falsas, obtendo uma sensibilidade de $94,1 \%$, especificidade em $73,1 \%$ e a uma acurácia de $90,3 \%$. O resultado menos significativo é do classificador $D e-$ cisionTable resultando no acerto de 124 imagens validada como corretas e 20 falsas, de modo a obter uma sensibilidade de $99,0 \%$, especificidade de 56,8 e uma acurácia $86,1 \%$.

A Tabela 4 mostra os resultados obtidos na classificação, aplicando o algoritmo kmeans com a região circular para segmentação junto com descritores de textura baseados em IDF para extrair as características. 
Tabela 4. Resultados da classificação com o k-means e a região circular.

\begin{tabular}{cccccccc}
\hline & FP & FN & VP & VN & Sensibilidade & Especificidade & Acurácia \\
\hline BayesNet & $\mathbf{3}$ & $\mathbf{7}$ & $\mathbf{1 1 5}$ & $\mathbf{1 9}$ & $\mathbf{9 4 , 3} \%$ & $\mathbf{8 8 , 9 \%}$ & $\mathbf{9 3 , 3} \%$ \\
J48 & 5 & 5 & 113 & 21 & $95,8 \%$ & $79,2 \%$ & $\mathbf{9 3 , 0 \%}$ \\
AttributeSelectedClassifier & 5 & 5 & 113 & 21 & $95,8 \%$ & $79,2 \%$ & $93,0 \%$ \\
DecisionTable & $\mathbf{3}$ & $\mathbf{9}$ & $\mathbf{1 1 5}$ & $\mathbf{1 7}$ & $\mathbf{9 2 , 7 \%}$ & $\mathbf{8 7 , 5 \%}$ & $\mathbf{9 1 , 9 \%}$ \\
\hline
\end{tabular}

Diante dos resultados apresentados na Tabela 4, o classificador BayesNet obteve o melhor resultado com uma porcentagem de sensibilidade com 94,3\%, especificidade em $88,9 \%$ e acurácia com $93,3 \%$, validando como corretas 134 imagens e 10 sendo falsas. $\mathrm{O}$ classificador que apresentou o pior resultado foi o DecisionTable, de maneira a obter uma sensibilidade em $92,7 \%$, especificidade de $87,5 \%$ e acurácia com $91,9 \%$, classificando 122 imagens como corretas e 22 sendo falsas.

A Tabela 5 mostra os resultados adquiridos através da junção de todas a características obtidas nos quatro métodos mostrados anteriormente.

Tabela 5. Resultados da classificação das características todos os métodos juntos.

\begin{tabular}{cccccccc}
\hline & FP & FN & VP & VN & Sensibilidade & Especificidade & Acurácia \\
\hline BayesNet & 33 & 30 & 439 & 74 & $93,6 \%$ & $69,2 \%$ & $89,1 \%$ \\
J48 & $\mathbf{3 3}$ & $\mathbf{3 3}$ & $\mathbf{4 3 9}$ & $\mathbf{7 1}$ & $\mathbf{9 3 , 0} \%$ & $\mathbf{6 8 , 3} \%$ & $\mathbf{8 8 , 5 \%}$ \\
AttributeSelectedClassifier & 46 & 20 & 426 & 84 & $95,5 \%$ & $64,6 \%$ & $\mathbf{8 8 , 5 \%}$ \\
DecisionTable & $\mathbf{3 3}$ & $\mathbf{3 0}$ & $\mathbf{4 3 9}$ & $\mathbf{7 4}$ & $\mathbf{9 3 , 6} \%$ & $\mathbf{6 9 , 2} \%$ & $\mathbf{8 9 , \mathbf { 1 } \%}$ \\
\hline
\end{tabular}

Na Tabela 5 o classificador que obteve a maior relevância nos resultados foi o DecisionTable apresentando uma sensibilidade de 93,6\%, especificidade de 69,2\% e uma acurácia em $89,1 \%$, sendo que, foram utilizadas 144 imagens, onde foram extraídas vinte características para cada imagem. O classificador que apresentou o pior resultado foi o $J 48$, apresentando uma sensibilidade em 93,0\%, especificidade de 68,3 e acurácia com $88,5 \%$.

A Tabela 6 apresenta os resultados da comparação dos trabalhos relacionados com a metodologia desenvolvida.

Tabela 6. Comparação da metodologia dos trabalhos relacionados.

\begin{tabular}{ccc}
\hline Trabalhos & Bases & Acurácia \% \\
\hline Santos e Veras (2012) & RIM-ONE & $87,5 \%$ \\
Claro (2015) & RIM-ONE & $93,67 \%$ \\
Santos et al. (2015) & RIO-ONE, DRISHTI--GS e DRIONS-DB & $83,0 \%$ \\
Pinheiro (2015) & DRISHTI & $91,75 \%$ \\
Metodologia & RIM-ONE & $\mathbf{9 7 , 9 \%}$ \\
\hline
\end{tabular}

A Tabela 6 mostra a porcentagem de acurácia dos trabalhos relacionados e da metodologia proposta, de modo, a analisar que a maior taxa de acurácia é da metodologia proposta obtendo 97,9\% utilizando a base RIM-ONE com 144 imagens saudáveis 
e com vários níveis de glaucoma. Os resultados apresentados na Tabela 6 mostram que em comparação com os demais trabalhos encontrados na literatura, o método proposto mostra-se promissor, pois, alcançou resultados bem significativos, e na maior parte dos casos, o melhor resultado. É importante salientar, que a comparação com os trabalhos é meramente subjetiva, pois os casos analisados variam, assim como as bases de imagens utilizadas, sendo estes, fatores primordiais para uma comparação fidedigna.

\section{Conclusão}

A partir dos resultados obtidos na classificação, pode-se concluir que, a metodologia proposta utilizando Otsu e k-means para segmentação do DO e os IDF na extração de características com descritores de textura apresentaram resultados expressivos. O método Otsu obteve melhores resultados que o método k-means, de forma a avaliar que o método Otsu foi mais eficiente para a detecção automática do glaucoma.

\section{Referências}

Andrade, E. O., Sampaio, I. G. B., Viterbo, J., Silva, J. M. M., e Boscarioli, C. (2016). Identificação de perfis de consumo domiciliar de energia elétrica a partir de algoritmos de agrupamento. XII Simpósio Brasileiro de Sistemas de Informação.

Bertholdo, F. A. R. (2007). Técnicas de limiarização para melhorar a qualidade visual de documentos históricos. Dissertação de Mestrado. Pós-Graduação em Ciência da Computação da Universidade Federal de Minas Gerais.

Claro, L. M. (2015). Detecção automática do glaucoma por extração de características e classificação. Curso de Sistemas de Informação da Universidade Federal do Piauí. Picos - PI, Brasil.

Mello, A. A. P., Susanna, J. R., e Almeida, H. G. (2016). Glaucoma. 4. ed. Rio de Janeiro: Cultura Médica. 512 p.

Moreira, J. A. (2014). Diferentes abordagens de análise da diversidade biológica da anurofauna do sul de minas gerais. Dissertação de Mestrado em Ecologia e Tecnologia Ambiental pela Universidade Federal de Alfenas - MG.

Oliveira, W. E. B. e Fernandes, S. R. (2016). Classificação de padrões em imagens usando descritores de textura. Bacharelado em Sistemas de Informação.

Pinheiro, A. F. C., Almeida, J. D. S., Junior, G. B., e Silva, A. C. (2015). Metodologia computacional para deteção automática do glaucoma em imagens de fundo de olho. Universidade Federal do Maranhão - São Luís - MA - Brasil.

Resnikoff, S., Pascolini, D., Etyále, D. E., Kocur, I., Pararajasegaram, R., Pokharel, G. P., e Mariotti, S. P. (2004). Global data on visual impairment in the year 2002. Bulletin of the World Health Organization.

Santos, L. M., Araújo, F. H. D., Claro, M. L., Silva, W. L., Silva, R. M. V., e Drumond, P. M. L. L. (2015). Implementação e comparação de um método de deteção e segmentação automática do disco óptico em diferentes bases de imagens da retina. Curso de Sistemas de Informação da Universidade Federal do Piauí. Picos - PI, Brasil.

Santos, L. M. R. e Veras, R. M. S. (2012). Levantamento, descrição e detecção dos principais atributos para o diagnóstico de glaucoma. Universidade Federal do Piauí UFPI, Brasil. 